\title{
Simple Waves characterizing wave propagation in a nonlinear elastic medium
}

\author{
Takao Momoi*
}

(Received July 14, 1998; Revised April 13, 1999; Accepted April 14, 1999)

\begin{abstract}
In the field of nonlinear waves, there exist two kinds of waves, i.e., one is Non-Coupled Simple Wave (nonlinear $\mathrm{P}$ wave) and Coupled Simple Wave (nonlinear S wave). In this paper, the problem is limitted to the two-dimensional case. After numerical computations, we have then found that the behavior of waves in the nonlinear wave field is governed by the theory of Simple Waves. Even complex wave form can be analyzed as composite Simple Waves.
\end{abstract}

\section{Introduction}

In the previous papers (Momoi, 1990, 1992; these papers will be referred to papers $\mathrm{A}$ and $\mathrm{B}$, respectively), we found two kinds of Simple Waves in a nonlinear elastic medium, that is to say, Non-Coupled Simple Wave (only a longitudinal component) and Coupled Simple Wave (a transverse component accompanied by a weak longitudinal component).

In order to clarify that these Simple Waves behave as dominant waves in nonlinear field, more intensive numerical computations was carried out in two-dimensonal case in this paper by use of the method in papers A and B. The behavior of the waves obtained is then explained by using the theory of Simple Waves.

\section{Simple Waves}

In papers $\mathrm{A}$ and $\mathrm{B}$, we have obtained expressions for Simple Waves. By use of second-order equations in twodimensional case under new notations, these Simple Waves are described here in the case of an isotropic elastic medium as follows.

Let $(x, z)$ be the components of Cartesian coordinates, $(u, w)$ the displacement components of Simple Waves in the longitudinal ( $x$-axis) and transverse ( $z$-axis) direction, respectively, $t$ a time variable, $v_{\mathrm{p}}$ and $v_{\mathrm{s}}$ the velocities of linear $\mathrm{P}$ and $\mathrm{S}$ waves, respectively, and $\{\lambda, \mu, A, B, C\}$ the elastic constants. Furthermore, these elastic coefficients are normalized by $\mu$ as follows.

$$
\begin{aligned}
& L_{m}=\lambda / \mu, A_{m}=A / \mu, \\
& B_{m}=B / \mu, C_{m}=C / \mu .
\end{aligned}
$$

(i) Non-Coupled Simple Wave

$$
\begin{aligned}
& u=\left(2 k_{r 1} V_{\mathrm{p} 1}\right) / g_{1}, \quad w=0, \\
& V_{\mathrm{p} 1}=\left(v_{\mathrm{p}}^{2}-v_{r 1}^{2}\right) / v_{\mathrm{s}}^{2}, k_{r 1}=v_{r 1} t-x, \\
& g_{1}=6+2 A_{m}+6 B_{m}+2 C_{m}+3 L_{m},
\end{aligned}
$$

where $v_{r 1}$ is a velocity of Non-Coupled Simple Wave.

*2-25-3, Shakujiidai, Nerimaku, Tokyo, 177-0045, Japan.

Copy right (C) The Society of Geomagnetism and Earth, Planetary and Space Sciences (SGEPSS); The Seismological Society of Japan; The Volcanological Society of Japan; The Geodetic Society of Japan; The Japanese Society for Planetary Sciences. (ii) Coupled Simple Wave

$$
\begin{aligned}
& u=\left(k_{r 2} V_{\mathrm{s} 2}\right) / f_{1}, \\
& w=s g_{w}\left(k_{r 2} W_{r}^{1 / 2}\right) /\left(f_{1} v_{\mathrm{s}}\right), \\
& W_{r}=V_{\mathrm{s} 2}\left(2 v_{\mathrm{p}}^{2}+v_{\mathrm{s}}^{2}\left(-2+\left(2-g_{1} / f_{1}\right) V_{\mathrm{s} 2}\right)\right), \\
& V_{\mathrm{s} 2}=1-v_{r 2}^{2} / v_{\mathrm{s}}^{2}, k_{r 2}=v_{r 2} t-x, \\
& f_{1}=2+A_{m} / 2+B_{m}+L_{m},
\end{aligned}
$$

where $v_{r 2}$ is a velocity of Coupled Simple Wave and $s g_{w}$ a double sign \pm associated with the displacement $w$.

\section{Finite-Difference Equation}

Let $h$ be a unit length of the coordinate axes $x$ and $z$. By use of the following expressions of time, coordinates and displacement components normalized by $h$,

$$
\begin{aligned}
& \hat{t}=h \cdot v_{\mathrm{p}} t, \quad \hat{x}=h \cdot x, \quad \hat{z}=h \cdot z, \\
& \hat{u}=h \cdot u, \quad \hat{w}=h \cdot w .
\end{aligned}
$$

The governing equation is given by (refer to papers A and B)

$$
\begin{aligned}
& \partial \hat{u} / \partial \hat{t}=U, \quad \partial \hat{w} / \partial \hat{t}=W \\
& \partial U / \partial \hat{t}=T_{10}+T_{11}, \quad \partial W / \partial \hat{t}=T_{30}+T_{31}
\end{aligned}
$$

with

$$
\begin{aligned}
& T_{10}=\hat{u}_{\hat{x} 2}+\hat{w}_{\hat{x} \hat{z}} L_{A M}+\left(\hat{u}_{\hat{z} 2} v_{\mathrm{s}}^{2}\right) / v_{\mathrm{p}}^{2}, \\
& T_{30}=\hat{w}_{\hat{z} 2}+\hat{u}_{\hat{x} \hat{z}} L_{A M}+\left(\hat{w}_{\hat{x} 2} v_{\mathrm{s}}^{2}\right) / v_{\mathrm{p}}^{2},
\end{aligned}
$$

and

$$
\begin{aligned}
T_{11}= & \left(g_{1} \hat{u}_{\hat{x}} \hat{u}_{\hat{x} 2}+2 f_{1} \hat{u}_{\hat{x} \hat{z}} \hat{u}_{\hat{z}}+f_{1} \hat{u}_{\hat{x}} \hat{u}_{\hat{z} 2}\right. \\
& +2 f_{2} \hat{u}_{\hat{x} \hat{z}} \hat{w}_{\hat{x}}+f_{2} \hat{u}_{\hat{z}} \hat{w}_{\hat{x} 2}+f_{1} \hat{w}_{\hat{x}} \hat{w}_{\hat{x} 2} \\
& +f_{3} \hat{u}_{\hat{x}} \hat{w}_{\hat{x} \hat{z}}+g_{2} \hat{u}_{\hat{x} 2} \hat{w}_{\hat{z}}+f_{1} \hat{u}_{\hat{z} 2} \hat{w}_{\hat{z}} \\
& \left.+f_{3} \hat{w}_{\hat{x} \hat{z}} \hat{w}_{\hat{z}}+f_{1} \hat{u}_{\hat{z}} \hat{w}_{\hat{z} 2}+f_{2} \hat{w}_{\hat{x}} \hat{w}_{\hat{z} 2}\right)\left(v_{\mathrm{s}} / v_{\mathrm{p}}\right)^{2}, \\
T_{31}= & \left(f_{3} \hat{u}_{\hat{x}} \hat{u}_{\hat{x} \hat{z}}+f_{2} \hat{u}_{\hat{x} 2} \hat{u}_{\hat{z}}+f_{1} \hat{u}_{\hat{z}} \hat{u}_{\hat{z} 2}\right. \\
& +f_{1} \hat{u}_{\hat{x} 2} \hat{w}_{\hat{x}}+f_{2} \hat{u}_{\hat{z} 2} \hat{w}_{\hat{x}}+f_{1} \hat{u}_{\hat{x}} \hat{w}_{\hat{x} 2} \\
& +2 f_{2} \hat{u}_{\hat{z}} \hat{w}_{\hat{x} \hat{z}}+2 f_{1} \hat{w}_{\hat{x}} \hat{w}_{\hat{x} \hat{z}}+f_{3} \hat{u}_{\hat{x} \hat{z}} \hat{w}_{\hat{z}} \\
& \left.+f_{1} \hat{w}_{\hat{x} 2} \hat{w}_{\hat{z}}+g_{2} \hat{u}_{\hat{x}} \hat{w}_{\hat{z} 2}+g_{1} \hat{w}_{\hat{z}} \hat{w}_{\hat{z} 2}\right)\left(v_{\mathrm{s}} / v_{\mathrm{p}}\right)^{2},
\end{aligned}
$$


where

$$
\begin{aligned}
& L_{A M}=1-\left(v_{s} / v_{p}\right)^{2}, \quad f_{2}=1+A_{m} / 2+B_{m}, \\
& g_{2}=2 B_{m}+2 C_{m}+L_{m}, \quad f_{3}=f_{2}+g_{2}, \\
& \xi_{\hat{x}}=\partial \xi / \partial \hat{x}, \quad \xi_{\hat{x} 2}=\partial^{2} \xi / \partial \hat{x}^{2}, \\
& \xi_{\hat{z}}=\partial \xi / \partial \hat{z}, \quad \xi_{\hat{z} 2}=\partial^{2} \xi / \partial \hat{z}^{2}, \\
& \xi_{\hat{x} \hat{z}}=\partial^{2} \xi / \partial \hat{x} \partial \hat{z} . \quad(\text { for } \xi=\hat{u}, \hat{w}, U \text { and } W)
\end{aligned}
$$

In order to solve the above equation by use of finitedifferenece method, $(\hat{u}, \hat{w})$ and $(U, W)$ are expanded by Taylor expansion up to the terms of second order with respect to time step $d \hat{t}\left(=\hat{t}-\hat{t}_{0}\right)$ (refer to papers A and B).

$$
\begin{aligned}
& \hat{u}=\hat{u}_{0}+d \hat{u}_{1} d \hat{t}+d \hat{u}_{2} d \hat{t}^{2} / 2, \\
& \hat{w}=\hat{w}_{0}+d \hat{w}_{1} d \hat{t}+d \hat{w}_{2} d \hat{t}^{2} / 2, \\
& U=U_{0}+d U_{1} d \hat{t}+d U_{2} d \hat{t}^{2} / 2, \\
& W=W_{0}+d W_{1} d \hat{t}+d W_{2} d \hat{t}^{2} / 2,
\end{aligned}
$$

with

$$
\begin{gathered}
d \Theta_{1}=(\partial \Theta / \partial \hat{t})_{0}, d \Theta_{2}=\left(\partial^{2} \Theta / \partial \hat{t}^{2}\right)_{0}, \\
(\text { for } \Theta=\hat{u}, \hat{w}, U \text { and } W)
\end{gathered}
$$

where $(\hat{u}, \hat{w}, U, W)$ and $\left(\hat{u}_{0}, \hat{w}_{0}, U_{0}, W_{0}\right)$ are displacement and velocity components at a time $\hat{t}$ and $\hat{t}_{0}$, respectively, and other quantities with suffix 0 indicate the quantities at a time $\hat{t}_{0}$. The above method based on second-order expansion of Taylor series is much more stable and effective in convergence, so that the required time by this method is about a tenth of that by usual first-order finite-difference method.

After some reduction, the coefficients in (5) are expressed as

$$
\begin{aligned}
& d \hat{u}_{1}=U_{0}, \quad d \hat{w}_{1}=W_{0}, \\
& d \hat{u}_{2}=(\partial U / \partial \hat{t})_{0}=\left(T_{10}+T_{11}\right)_{0}, \\
& d \hat{w}_{2}=(\partial W / \partial \hat{t})_{0}=\left(T_{30}+T_{31}\right)_{0}, \\
& d U_{1}=(\partial U / \partial \hat{t})_{0}=\left(T_{10}+T_{11}\right)_{0}, \\
& d W_{1}=(\partial W / \partial \hat{t})_{0}=\left(T_{30}+T_{31}\right)_{0}, \\
& d U_{2}=\left(\partial^{2} U / \partial \hat{t}\right)_{0}=\left(T_{10 t}+T_{11 t}\right)_{0}, \\
& d W_{2}=\left(\partial^{2} W / \partial \hat{t}\right)_{0}=\left(T_{30 t}+T_{31 t}\right)_{0},
\end{aligned}
$$

with

$$
\begin{aligned}
T_{10 t}= & \partial T_{10} / \partial \hat{t}, \\
= & U_{\hat{x} 2}+W_{\hat{x} \hat{z}} L_{A M}+U_{\hat{z} 2}\left(v_{\mathrm{s}} / v_{\mathrm{p}}\right)^{2}, \\
T_{30 t}= & \partial T_{30} / \partial \hat{t}, \\
= & W_{\hat{z} 2}+U_{\hat{x} \hat{z}} L_{A M}+W_{\hat{x} 2}\left(v_{\mathrm{s}} / v_{\mathrm{p}}\right)^{2}, \\
T_{11 t}= & Q_{1 t}\left(v_{\mathrm{s}} / v_{\mathrm{p}}\right)^{2}, \quad T_{31 t}=Q_{3 t}\left(v_{\mathrm{s}} / v_{\mathrm{p}}\right)^{2}, \\
Q_{1 t}= & g_{1} \hat{u}_{\hat{x} 2} U_{\hat{x}}+f_{1} \hat{u}_{\hat{z} 2} U_{\hat{x}}+f_{3} \hat{w}_{\hat{x} \hat{z}} U_{\hat{x}} \\
& +g_{1} \hat{u}_{\hat{x}} U_{\hat{x} 2}+g_{2} \hat{w}_{\hat{z}} U_{\hat{x} 2}+2 f_{1} \hat{u}_{\hat{z}} U_{\hat{x} \hat{z}} \\
& +2 f_{2} \hat{w}_{\hat{x}} U_{\hat{x} \hat{z}}+2 f_{1} \hat{u}_{\hat{x} \hat{z}} U_{\hat{z}}+f_{2} \hat{w}_{\hat{x} 2} U_{\hat{z}} \\
& +f_{1} \hat{w}_{\hat{z} 2} U_{\hat{z}}+f_{1} \hat{u}_{\hat{x}} U_{\hat{z} 2}+f_{1} \hat{w}_{\hat{z}} U_{\hat{z} 2} \\
& +2 f_{2} \hat{u}_{\hat{x} \hat{z}} W_{\hat{x}}+f_{1} \hat{w}_{\hat{x} 2} W_{\hat{x}}+f_{2} \hat{w}_{\hat{z} 2} W_{\hat{x}} \\
& +f_{2} \hat{u}_{\hat{z}} W_{\hat{x} 2}+f_{1} \hat{w}_{\hat{x}} W_{\hat{x} 2}+f_{3} \hat{u}_{\hat{x}} W_{\hat{x} \hat{z}} \\
& +f_{3} \hat{w}_{\hat{z}} W_{\hat{x} \hat{z}}+g_{2} \hat{u}_{\hat{x} 2} W_{\hat{z}}+f_{1} \hat{u}_{\hat{z} 2} W_{\hat{z}} \\
& +f_{3} \hat{w}_{\hat{x} \hat{z}} W_{\hat{z}}+f_{1} \hat{u}_{\hat{z}} W_{\hat{z} 2}+f_{2} \hat{w}_{\hat{x}} W_{\hat{z} 2},
\end{aligned}
$$

$$
\begin{aligned}
Q_{3 t}= & f_{3} \hat{u}_{\hat{x} \hat{z}} U_{\hat{x}}+f_{1} \hat{w}_{\hat{x} 2} U_{\hat{x}}+g_{2} \hat{w}_{\hat{z} 2} U_{\hat{x}} \\
& +f_{2} \hat{u}_{\hat{z}} U_{\hat{x} 2}+f_{1} \hat{w}_{\hat{x}} U_{\hat{x} 2}+f_{3} \hat{u}_{\hat{x}} U_{\hat{x} \hat{z}} \\
& +f_{3} \hat{w}_{\hat{z}} U_{\hat{x} \hat{z}}+f_{2} \hat{u}_{\hat{x} 2} U_{\hat{z}}+f_{1} \hat{u}_{\hat{z} 2} U_{\hat{z}} \\
& +2 f_{2} \hat{w}_{\hat{x} \hat{z}} U_{\hat{z}}+f_{1} \hat{u}_{\hat{z}} U_{\hat{z} 2}+f_{2} \hat{w}_{\hat{x}} U_{\hat{z} 2} \\
& +f_{1} \hat{u}_{\hat{x} 2} W_{\hat{x}}+f_{2} \hat{u}_{\hat{z} 2} W_{\hat{x}}+2 f_{1} \hat{w}_{\hat{x} \hat{z}} W_{\hat{x}} \\
& +f_{1} \hat{u}_{\hat{x}} W_{\hat{x} 2}+f_{1} \hat{w}_{\hat{z}} W_{\hat{x} 2}+2 f_{2} \hat{u}_{\hat{z}} W_{\hat{x} \hat{z}} \\
& +2 f_{1} \hat{w}_{\hat{x}} W_{\hat{x} \hat{z}}+f_{3} \hat{u}_{\hat{x} \hat{z}} W_{\hat{z}}+f_{1} \hat{w}_{\hat{x} 2} W_{\hat{z}} \\
& +g_{1} \hat{w}_{\hat{z} 2} W_{\hat{z}}+g_{2} \hat{u}_{\hat{x}} W_{\hat{z} 2}+g_{1} \hat{w}_{\hat{z}} W_{\hat{z} 2} .
\end{aligned}
$$

In the above, $\hat{u}, \hat{w}, U$ and $W$ with suffix indicate the derivatives given by (4) and these are replaced by the following difference equation.

$$
\begin{aligned}
& \xi_{\hat{x}}=\left(\xi_{21}-\xi_{01}\right) /(2 \delta h), \quad \xi_{\hat{z}}=\left(\xi_{12}-\xi_{10}\right) /(2 \delta h), \\
& \xi_{\hat{x} 2}=\left(\xi_{01}+\xi_{21}-2 \xi_{11}\right) / \delta h^{2}, \\
& \xi_{\hat{z} 2}=\left(\xi_{10}+\xi_{12}-2 \xi_{11}\right) / \delta h^{2}, \\
& \xi_{\hat{x} \hat{z}}=\left(\xi_{00}+\xi_{22}-\xi_{02}-\xi_{20}\right) /\left(4 \delta h^{2}\right),
\end{aligned}
$$

where the numerical suffix in the above indicates a mesh point described in Fig. 1 and $\delta h$ is a mesh interval normalized by $h$.

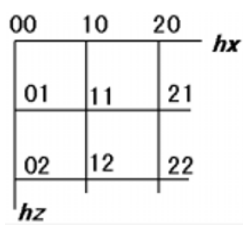

Fig. 1. Mesh points, where $h x$ and $h z$ indicate $\hat{x}$ and $\hat{z}$, respectively.

A stability problem of the computation always occurrs in the numerical computation by use of the finite difference method. On the occasion of the numerical computation in the linear equation, we can analytically find a stability criterion like Neuman condition. In the case of nonlinear equation, such a treatment is impossible, so that the stabilty of the computation is confirmed by a lot of numerical trials of the computation. After such trials, the following values

$$
d \hat{t}=0.1, \quad \delta h=0.5
$$

are confirmed to keep the numerical computation sufficiently stable.

\section{Initial Condition}

The following expression for an initial condition is given near the wave origin.

$$
\begin{aligned}
\xi= & (A(\xi) / 2)\{1+\cos (\hat{x} \pi / 4)\}, \\
& (-4<\hat{x}<4)(\text { for } \xi=\hat{u}, \hat{w}, U \text { and } W) .
\end{aligned}
$$

Since we are treating the problem in two-dimension, a wave origin is like a mountain ridge.

\section{Numerical Experiment}

The ability of a computer goes up more than that when the computation in papers A and B was carried out, so that new computation was made by use of more efficient computer. Then the values of elastic coefficients were specified as

$$
L_{m}=A_{m}=B_{m}=C_{m}=1.0 .
$$




\subsection{Instance 1}

In Fig. 2, the amplitudes in (6) are expressed as

$$
A(\hat{u})=0.2 \text { and } A(\xi)=0(\xi=\hat{w}, U, W) .
$$

The wave origin (6) with the above amplitude indicates that a push-type displacement is given at the origin in the direction of the wave propagation.

As found from Fig. 2, the positive side (push part) of the amplitude of the wave decreses with propagation, while, in due course, the negative side (pull part) increases. Each wave form of these waves is a pulse of triangular type instead of a sinusoidal one, and further propagated at a velocity near linear $\mathrm{P}$ wave (not exact $\mathrm{P}$ wave velocity). The above exposed results are very important together with those in the next numerical instance.

In the figure, $h x$ and $h u$-axes indicate normalized distance $h x=\hat{x}$ and normalized displacement multiplied with 'Vertical_scale' (described on the top of the figure), i.e., $h u=($ Vertical_scale $) \times \hat{u}$. The first (leftmost) broken line is that at $\hat{t}=0$ (initial value). The other broken lines indicate wave forms by step time 50 and the bold solid line a final wave form, which is that at $\hat{t}=500$ in this case, respectively. These conventions except the last one associated with the time of the last wave form follow in the same way in the subsequent Figs. 3, 5, 6, 7 and 8.

In Fig. 3, the amplitudes in (6) are expressed as

$$
A(\hat{u})=-0.2 \text { and } A(\xi)=0(\xi=\hat{w}, U, W) .
$$

The wave origin (6) with the above amplitude indicates that a pull-type displacement is given at the origin in the direction of the wave propagation.

As found from Fig. 3, the pull-type wave at the wave origin is propagated by holding a pull-type feature at a velocity

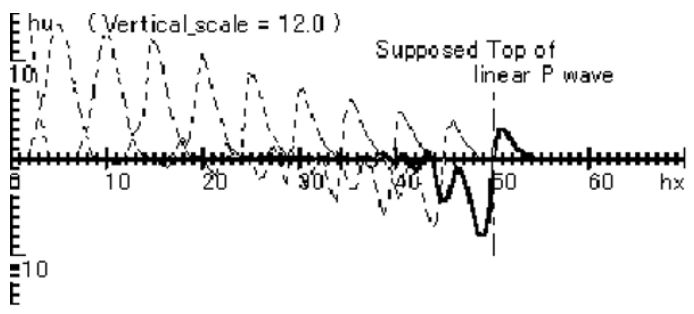

Fig. 2. Propagation of Non-Coupled Simple Wave in the case of positive initial displacement of wave origin.

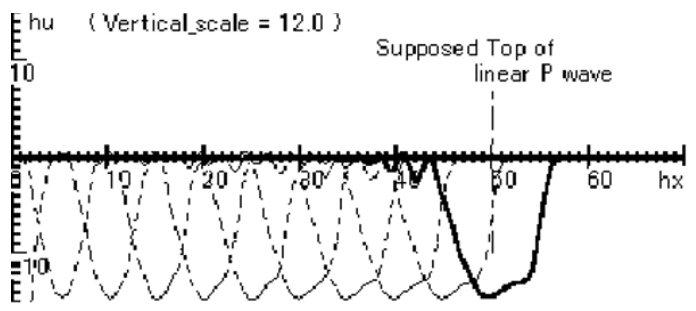

Fig. 3. Propagation of Non-Coupled Simple Wave in the case of negative initial displacement of wave origin, where $h x=\hat{x}$ and $h u=($ Vertical_scale $) \times \hat{u}$. The final wave form (bold line) is that at $\hat{t}=500$. near linear $\mathrm{P}$ wave. As propagating, the width of the wave becomes wider by changing the wave form into a trapezoidal one. The wave shown in Figs. 2 and 3 will be named nonlinear $\mathrm{P}$ wave later on.

Let us explain the above-mentioned two features such that the push-type nonlinear P wave pulse (Fig. 2) becomes narrower in a triangular form and the pull-type nonlinear $\mathrm{P}$ wave pulse (Fig. 3) becomes wider in a trapezoidal form.

We assume that these waves consist of a few fragments of Simple Waves described in Section 2. Though we use a term of 'assume' in here, all numerical experiments indicate that this assumption is not only an assumption, but a truth itself.

Suppose that Non-Coupled Simple (nonlinear P) Waves in Section 2 constitute the waves exposed in Figs. 2 and 3. We can put

$$
v_{r 1}=v_{\mathrm{p}}+d v_{\mathrm{p}}\left(d v_{\mathrm{p}}: \text { a small quantity }\right)
$$

in (1). By taking the term up to first order of $d v_{\mathrm{p}}$, we obtain

$$
u=\left(-4 d v_{\mathrm{p}} v_{\mathrm{p}}\left(v_{r 1} t-x\right)\right) /\left(g_{1} v_{\mathrm{s}}^{2}\right)
$$

The expressions (7) and (8) indicate very important facts such that

(i) when the wave front (fragment of Non-Coupled Simple Wave) is propagated at a velocity faster than that of linear $\mathrm{P}$ wave $\left(d v_{\mathrm{p}}>0\right)$, the wave front is of a pull-type ( $u$ decreases).

(ii) when the wave front is propagated at a velocity slower than that of linear $\mathrm{P}$ wave $\left(d v_{\mathrm{p}}<0\right)$, the wave front is of a push-type ( $u$ increases).

Let us consider a triangular or trapezoidal pulse of a wave composed of two push and pull Non-Coupled Simple Waves. As shown in Fig. 4, very interesting features are found from (i) and (ii) in (9). In this figure, lines with arrow Slow or Fast indicate wave fronts (Simple Waves) of a pulse which moves at a velocity slower or faster than that of linear $\mathrm{P}$ wave, respectively.

Two features in (9) leads to very interesting features such that

(i) a triangular push pulse (top figure) becomes narrower with the propagation,

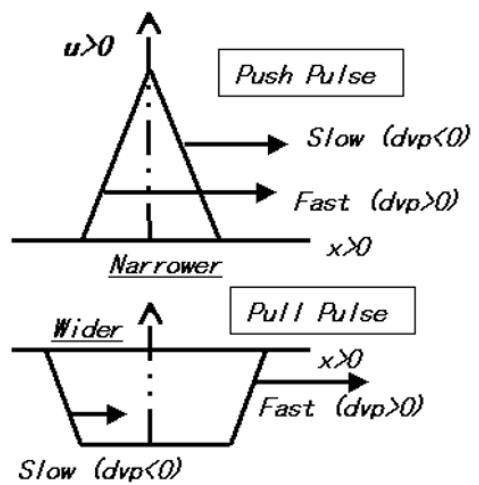

Fig. 4. Behavior of push- and pull-type pulse constituted by composite Simple Waves. $d v p$ indicates $d v_{\mathrm{p}}$. 
(ii) a triangular pull pulse (bottom figure) becomes wider with the propagation and tends to a trapezoidal form.

By use of the theoretical model mentioned above, the result of numerical experiments (Figs. 2 and 3) will be elucidated again. The phenomenon such that the push wave becomes smaller in a triangular form with the propagation (Fig. 2) can be explained by the top model in Fig. 4, while the phenomenon such that the pull wave becomes wider in a trapezoidal form with the propagation (Fig. 3) can be explained by the bottom model in Fig. 4 .

In the linear theory, a trapezoidal wave like that in Fig. 3 is named a saturated wave. On the occasion of the nonlinear theory, the term saturated wave is not appropriate, since the generation process of the trapezoidal wave is completely irrespective of a saturation process.

Let us make mention of the advancing velocity of Coupled Simple Wave in here. Like the case of Non-Coupled Simple Wave, we put

$$
v_{r 2}=v_{\mathrm{s}}+d v_{\mathrm{s}}\left(d v_{\mathrm{s}}: \text { a small quantity }\right)
$$

Substituting the above into (2) and taking the term up to first-order of $d v_{\mathrm{s}}$, we have

$$
\begin{gathered}
u=-2 d v_{\mathrm{s}}\left(v_{r 2} t-x\right) /\left(f_{1} v_{\mathrm{s}}\right), \\
w=s g_{w} 2\left(-d v_{\mathrm{s}}\right)^{1 / 2}\left(v_{\mathrm{p}}^{2}-v_{\mathrm{s}}^{2}\right)^{1 / 2} \\
\cdot\left(v_{r 2} t-x\right) /\left(f_{1} v_{\mathrm{s}}^{3 / 2}\right),
\end{gathered}
$$

In the above expression, the displacement component $w$ has a factor $\left(-d v_{\mathrm{s}}\right)^{1 / 2}$, so that $d v_{\mathrm{s}}$ is required to be negative in order for the component $w$ to exist (square root is required to be real). In due course, Coupled Simple Wave is always propagated at a velocity less than that of linear $\mathrm{S}$ wave from (11). Furthermore, the longitudinal $u$-component $\left(\sim\left|d v_{\mathrm{s}}\right|\right)$ is smaller in quantitiy than the transverse $w$-component $(\sim$ $\left.\left|d v_{\mathrm{s}}\right|^{1 / 2}\right)$.

Some comment is made, in here, on the wave form like that appeared in Fig. 2. Such an oscillatory wave form is frequently found on the occasion of large earthquake. If the interpretation of the wave form like that is done based on a linear theory, the wave source is interpreted to be of an oscillatory type. If a nonlinear process occurs both near the wave source and during the propagation process, this interpretation based on a linear theory is completely wrong from the result in Fig. 2.

\subsection{Instance 2}

Figure 5 is an instance where the amplitude in the expression (6) is given by

$$
A(U)=0.2 \text { and } A(\xi)=0(\xi=\hat{u}, \hat{w}, W) .
$$

This expression indicates the case where a push velocity is given at the wave origin in the direction of the wave propagation. In this case, a push-type step wave appears in the figure, the height of which decreases gradually with the propagation. The front of the generated wave is a push-type Simple Wave and the top of the wave is propagated at a velocity slower than that of linear P wave as expected from (ii)

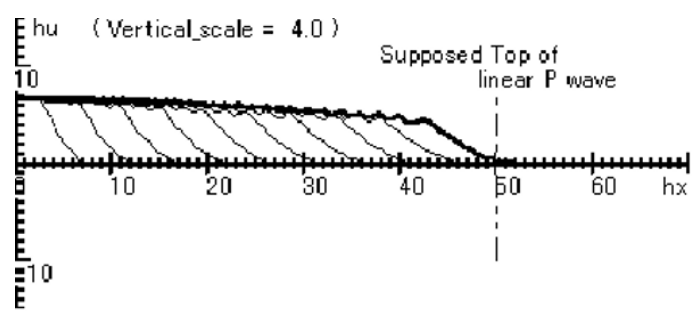

Fig. 5. Propagation of Non-Coupled Simple Wave in the case of positive initial velocity of wave origin, where $h x=\hat{x}$ and $h u=($ Vertical_scale $) \times \hat{u}$. The final wave form (bold line) is that at $\hat{t}=500$.

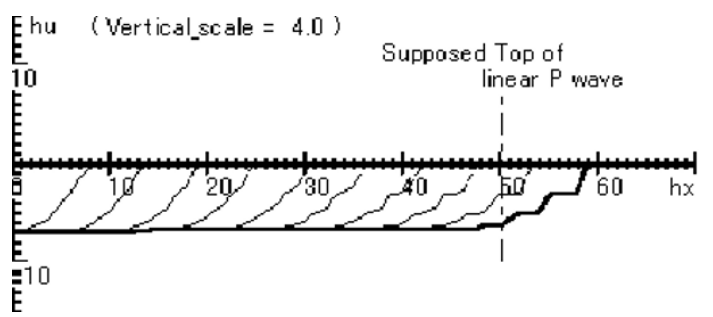

Fig. 6. Propagation of Non-Coupled Simple Wave in the case of negative initial velocity of wave origin, where $h x=\hat{x}$ and $h u=($ Vertical_scale $) \times \hat{u}$. The final wave form (bold line) is that at $\hat{t}=500$.

in (9). In the figure, 'Supposed Top ...' indicates an apex of a linear triangular $\mathrm{P}$ wave instead of a rightward foremost head.

Figure 6 is an instance where the amplitude in the expression (6) is given by

$$
A(U)=-0.2 \text { and } A(\xi)=0(\xi=\hat{u}, \hat{w}, W) .
$$

This expression indicates the case where a pull velocity is given at the wave origin in the direction of the wave propagation. In this case, a pull-type step wave appears in the figure. The wave front is a pull-type Simple Wave and the top of the wave is propagated at a velocity faster than that of linear P wave as expected from (i) in (9). In the part of the wave front, the dispersion of the wave (several steps) occurs in such a way that the foremost head part (steep gradient) is more accelerated than the later part (gentle gradient). This feature is explained as follows.

From (7) and (8), it is found that, if the gradient of $u$ is steeper (larger), $d v_{\mathrm{p}}$ is also larger (from (8)), so that the velocity of the wave becomes faster (from (7)).

\subsection{Instance 3}

Figure 7 is an instance where the amplitude in the expression (6) is given by

$$
A(\hat{w})=0.2 \text { and } A(\xi)=0(\xi=\hat{u}, U, W) .
$$

This expression indicates the case where an initial displacement is given at the wave origin in the transverse direction of the wave propagation. In this case, an triangular pulse appears as an composite wave of Coupled Simple Wave in the figure. In this figure, 'Supposed Top ...' indicates an apex of a triangular pulse of linear $\mathrm{S}$ wave instead of a foremost head. 


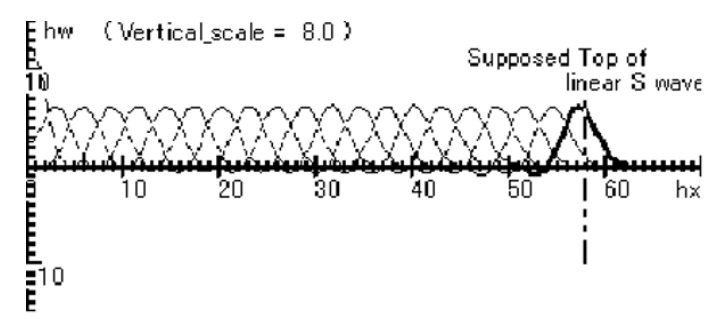

Fig. 7. Propagation of Coupled Simple Wave in the case of positive initial displacement of wave origin in the transverse direction, where $h x=\hat{x}$ and $h w=($ Vertical_scale $) \times \hat{w}$. The final wave form (bold line) is that at $\hat{t}=1000$.

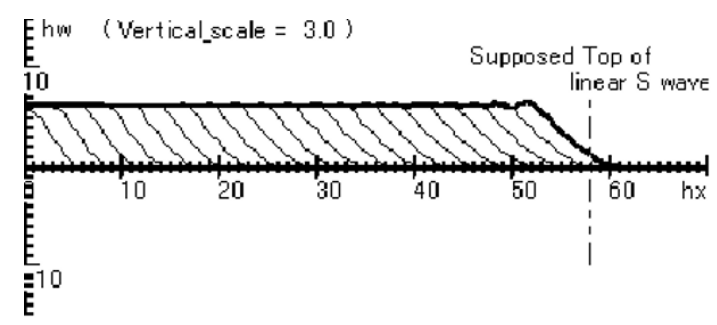

Fig. 8. Propagation of Coupled Simple Wave in the case of positive initial velocity of wave origin in the transverse direction, where $h x=\hat{x}$ and $h w=($ Vertical_scale $) \times \hat{w}$. The final wave form (bold line) is that at $\hat{t}=1000$.

Figure 8 is an instance where the amplitude in the expression (6) is given by

$$
A(W)=0.2 \text { and } A(\xi)=0(\xi=\hat{u}, \hat{w}, U) .
$$

This expression indicates the case where an initial velocity is given at the wave origin in the transverse direction of the wave propagation. In this case, a wave front of Coupled Simple Wave appears at the head of the wave.

In Figs. 7 and 8, the top of Coupled Simple Wave is propagated at a velocity a little slower than that of linear $\mathrm{S}$ wave as discussed in (11) and (12), so that Coupled Simple Wave is named nonlinear $\mathrm{S}$ wave.

\section{Conclusion}

In this study, we have found a very impotant fact such that the propagation of nonlinear waves is fundamentally governed by the theory of Simple Waves. Even oscillatory complex waves can be analyzed as waves consisting of several fragments of Simple Waves. This fact has an important significance in such a way that on the occasion of the response evaluation of nonlinear waves at the boundary we can assume, as the first approximation, the incident and reflected nonlinear waves to be a congregation of Simple Waves. By using nonlinear stress boundary conditions and the above Simple Waves, we can analyze the temporary behavior of nonlinear waves near the boundary at a certain moment.

Another important exposed fact is such that nonlinear push $P$ pulse becomes narrower in a triangular form with the propagation, while nonlinear pull $\mathrm{P}$ pulse becomes wider in a trapezoidal form with the propagation.

\section{References}

Momoi, T., Wave propagation in nonlinear-elastic isotropic media, Bull. Earthq. Res. Inst., 65, 413-432, 1990.

Momoi, T., The polarization of waves in an anisotropic nonlinear-elastic medium, Bull. Earthq. Res. Inst., 67, 1-20, 1992.

T. Momoi (e-mail: qyz1932@pb.highway.ne.jp) 